\title{
A ÍNSULA E O CONCEITO DE BLOCO CEREBRAL CENTRAL
}

\author{
Guilherme Carvalhal Ribas', Evandro de Oliveira²
}

\begin{abstract}
RESUMO - A caracterização anatômica de regiões encefálicas topograficamente bem definidas é particularmente útil para a prática neurocirúrgica por propiciar melhor compreensão da tridimensionalidade das suas estruturas e das lesões que as acometem, e por incitar uma maior sistematização dos seus acessos cirúrgicos. Neste sentido, se destaca no interior de cada hemisfério cerebral, um verdadeiro bloco único composto externamente pela ínsula, internamente pelos núcleos da base e tálamo, e que abriga no seu interior a cápsula interna. Com uma conformação predominantemente biconvexa e disposto entre a cisterna silviana e as cavidades ventriculares supratentoriais, esse bloco cerebral central morfologicamente se caracteriza como uma cabeça de cada metade do tronco encefálico, encoberta por todo o manto neocortical do seu hemisfério ao qual se une através de verdadeiros istmos constituídos pelos prolongamentos das diferentes partes da cápsula interna. Anteriormente e sob o sulco limitante anterior da ínsula se dispõem as fibras que compõem o ramo anterior da cápsula interna, superiormente e sob o seu sulco limitante superior se dispõem as fibras restantes do ramo anterior e as do joelho e do ramo posterior, entre as quais se destacam em importância funcional as fibras piramidais córtico-espinhais, e sob o sulco limitante inferior da ínsula se dispõem as partes retro e sub-lentiformes da cápsula interna, que englobam as radiações auditiva e visual. Lateralmente o bloco cerebral central tem a ínsula como um verdadeiro escudo externo das principais estruturas cerebrais subcorticais. As escolhas dos acessos microneurocirúrgicos às lesões relacionadas com o bloco cerebral central devem ter como maior preocupação as suas relações topográficas com o tálamo e com as fibras da cápsula interna.
\end{abstract}

PALAVRAS-CHAVE: ínsula, núcleos da base, tálamo, cápsula interna, neuroanatomia, microcirurgia.

\section{The insula and the central core concept}

ABSTRACT - The caracterization of well defined and circumscribed brain regions is particularly useful for the neurosurgical practice once it enhances the tridimensional understanding of its structures and related lesions, and because it induces the development and the utilization of more standard microneurosurgical approaches. In this direction, it is noteworthy that each cerebral hemisphere harbors an evident central core constituted externally by the insula, internally by the basal ganglia and the thalamus, and with the internal capsule within. With a biconvex configuration when seen from above, and located between the sylvian cistern and the supratentorial ventricular cavities, morphologically this central core resembles a head of each brainstem half top, covered by the neocortical mantle of its hemisphere. The central core is attached to the rest of the cerebral hemisphere by isthmi constituted by the different internal capsule fibers. Anteriorly and under the anterior limiting sulcus of the insula there are fibers of the internal capsule anterior limb, superiorly and under the superior limiting sulcus there are the rest of the anterior limb fibers, and the knee and posterior limb fibers that harbors the corticonuclear and the corticospinal tracts, and inferiorly and under the insular inferior limiting sulcus there are the sub- and the retrolentiform internal capsule fibers that enclose the auditory and the optic radiations. Laterally the central core is composed by the insular surface that resembles a shield of the main cerebral subcortical structures. The options of microneurosurgical approaches to the central core related lesions should consider particularly their relationships with the thalamus and with the internal capsule fibers.

KEY WORDS: insula, basal ganglia, thalamus, internal capsule, neuroanatomy, microsurgery.

\footnotetext{
${ }^{1}$ Professor Livre-Docente e Coordenador do Setor de Neuroanatomia Aplicada da Disciplina de Topografia Estrutural Humana do Departamento de Cirurgia da Faculdade de Medicina da Universidade de São Paulo, São Paulo SP, Brasil (FMUSP); ${ }^{2}$ Professor e Chefe da Disciplina de Neurocirurgia da Faculdade de Ciências Médicas da Universidade Estadual de Campinas, Campinas SP, Brasil (UNICAMP).
}

Recebido 5 Junho 2006, recebido na forma final 31 Agosto 2006. Aceito 21 Outubro 2006.

Dr. Guilherme Carvalhal Ribas - Rua Prof. Eduardo Monteiro 567 - 05614-120 São Paulo SP - Brasil. E-mail: guilherme@ribas.med.br 
A neuroimagenologia e a microneurocirurgia modernas requerem a compreensão da anatomia das estruturas neurais intracranianas principalmente no que diz respeito à tridimensionalidade das estruturas, às relações topográficas existentes entre elas, e às suas relações com os espaços naturais que são constituídos pelos espaços preenchidos pelo líquido cerebrospinal ${ }^{1-27}$.

Uma vez que em exames de imagens as estruturas encefálicas são identificadas após o reconhecimento inicial dos espaços naturais que as contêm, e que os seus acessos microneurocirúrgicos são feitos, sempre que possível, através desses mesmos espaços, a caracterização anatômica de regiões topograficamente bem definidas se torna útil por propiciar uma compreensão mais dirigida e particularizada das suas estruturas com as suas respectivas eloqüências funcionais e das lesões que as acometem, e por incitar uma maior sistematização dos seus acessos cirúrgicos. Em relação às lesões passíveis de tratamento neurocirúrgico, é ainda interessante observar que diferentes patologias que acometem uma mesma região do sistema nervoso central o fazem com peculiaridades anatômicas específicas que freqüentemente implicam no emprego de diferentes estratégias cirúrgicas, o que reforça a necessidade do conhecimento detalhado de cada região em particular.

Neste sentido, conforme apontado inicialmente pelo autor sênior da presente publicação (Oliveira E), do ponto de vista topográfico a ínsula constitui um verdadeiro escudo externo de um bloco de estruturas cerebrais anatomicamente muito evidentemente bem definido, de posição central em cada hemisfério, e formado pelas principais estruturas cerebrais profundas.

No presente texto foi adotada a nomenclatura da atual Terminologia Anatômica Internacional ${ }^{1,2}$, que substituiu as versões anteriores da Nomina Anatomica. No entanto, na ausência de termos correspondentes, e/ou ao se tratar de termos com denominações notoriamente já consagrados na prática médica, foram utilizados termos não pertencentes à Terminologia Anatômica Internacional.

\section{CONSIDERAÇõES ANATÔMICAS}

Do ponto de vista topográfico a superfície da ínsula nitidamente se caracteriza como sendo o escudo externo de um verdadeiro bloco cerebral central anatomicamente muito bem definido, e constituído em cada hemisfério pela própria ínsula, pelos núcleos da base, tálamo e cápsula interna (Fig 1).

Disposto entre a cisterna silviana, as cisternas parapedunculares (cisternas crural, ambiens e quadrigê- mina), e as cavidades ventriculares supratentoriais, visto superiormente esse bloco cerebral central possui uma conformação biconvexa, apresenta como face lateral o córtex insular, como face medial as superfícies intraventriculares do núcleo caudado e do tálamo, e contém no seu interior as fibras que compõem a cápsula interna. Enquanto a metade anterior da ínsula corresponde internamente à cabeça do núcleo caudado, a sua metade posterior se relaciona topograficamente com o tálamo.

Como cada tálamo morfologicamente se caracteriza como sendo o topo de cada metade do tronco encefálico dadas as íntimas conexões e continuidade anatômica existentes entre os tálamos e a porção tegmentar do mesencéfalo, cada bloco cerebral central arquitetonicamente equivale a uma verdadeira cabeça do tronco encefálico, circundada pelos ventrículos e cisternas supratentoriais, e envolta pelo telencéfalo. A fissura corióidea, que também se dispõem em forma de $C$ entre cada tálamo e cada fórnix $^{8,11,21}$, constitui um importante espaço de comunicação entre os compartimentos ventriculares e cisternais relacionados com o bloco cerebral central.

A superfície insular é constituída do chamado mesocórtex, que anatomicamente se situa entre o alocórtex mais antigo e de topografia mais medial (amígdala, hipocampo) e o isocórtex filogeneticamente mais recente e de topografia mais lateral (neocórtex dos hemisférios cerebrais) ${ }^{10,18,23}$. Incrustada entre os lobos frontal e temporal de cada hemisfério e constituindo a base de cada cisterna silviana, a ínsula possui uma superfície anterior e uma superfície lateral que se encontram recobertas pelos seus respectivos opérculos (do latim operculum: cortina) ${ }^{5}$, que correspondem às regiões mais ulteriormente desenvolvidas dos hemisférios cerebrais. Enquanto a superfície insular anterior é encoberta pelo opérculo fronto-orbital (constituído pela porção posterior do giro orbital posterior e pela parte orbital do giro frontal inferior), a sua superfície lateral é recoberta superiormente pelo opérculo frontoparietal (partes triangular e opercular do giro frontal inferior, giro subcentral ou prega frontoparietal inferior, e parte superior do giro supramarginal), e inferiormente pelo opérculo temporal (giro temporal superior/pólo temporal, e porção inferior do giro supramarginal) ${ }^{15,26}$.

As superfícies operculares frontoparietal e temporal da superfície súpero-lateral do cérebro se justapõem delineando o aspecto mais superficial de toda a porção lateral da fissura silviana, e ao se invaginarem passam a constituir as respectivas superfícies operculares que se confrontam caracterizando a fis- 

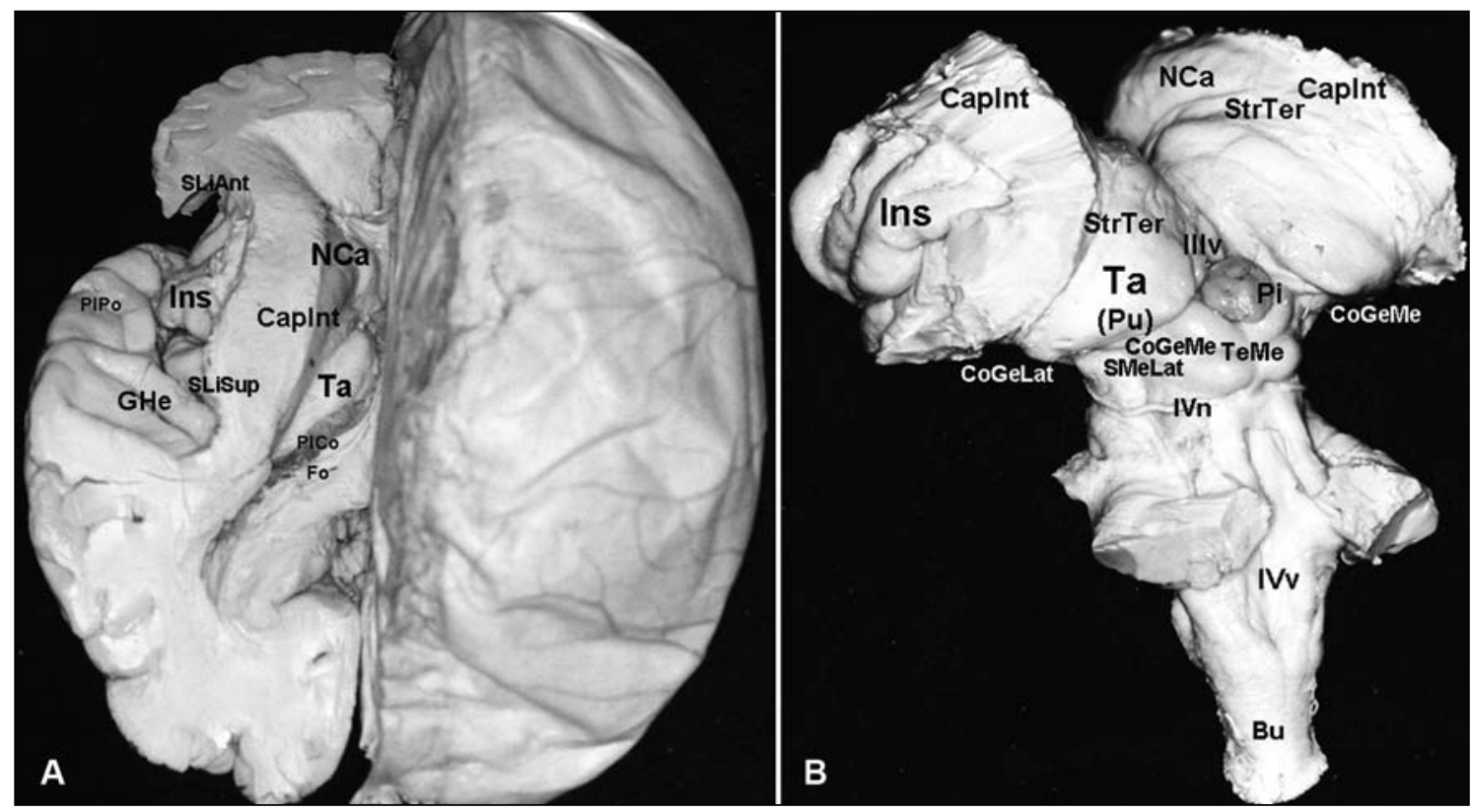

Fig 1. A ínsula e o bloco cerebral central. (A) a ínsula (Ins) corresponde a um verdadeiro escudo externo da cápsula interna (Caplnt), núcleos da base e tálamo (Ta), com os quais morfologicamente caracteriza um verdadeiro bloco central. (B) O bloco cerebral central morfologicamente se caracteriza como uma verdadeira cabeça disposta sobre cada metade do mesencéfalo e que se incorpora ao restante do hemisfério cerebral através de verdadeiros istmos constituídos pelas diferentes partes da cápsula interna (Caplnt) que se dispõem no seu interior. Bu: bulbo; Caplnt: cápsula interna; CoGeLat: corpo geniculado lateral; CoGeMe: corpo geniculado medial; Fo: fórnix; GHe: giro de Heschl; IIIv: III ventrículo; Ins: ínsula; IVn: nervo troclear; IVv: quarto ventrículo; NCa: núcleo caudado; Pi: glândula pineal; PICo: plexo corióideo; PIPo: plano polar; Pu: pulvinar do tálamo; SLiAnt: sulco limitante anterior; SLiSup: sulco limitante superior; SMeLat: sulco mesencefálico lateral; StrTer: estria terminal; Ta: tálamo; TeMe: tecto mesencefálico.

sura silviana propriamente dita, que por sua vez se amplia sobre a superfície insular e que abriga a cisterna silviana ${ }^{12,24,25,27}$. Enquanto a superfície opercular frontoparietal é predominantemente plana e perpendicular à convexidade cerebral, a superfície opercular temporal é dividida pelos giros transversos anterior e posterior de Heschl ${ }^{22,23} \mathrm{em}$ um plano anterior oblíquo que é denominado de plano polar e que efetivamente cobre a porção inferior da superfície insular lateral, e em um plano posterior perpendicular à convexidade denominado de plano temporal, de forma triangular e com vértice medial apontando para o átrio ventricular, e que se confronta com a superfície inferior da porção distal da superfície opercular frontoparietal.

A superfície lateral da ínsula se caracteriza como uma pirâmide de base triangular cujo vértice ânteroinferior constitui o limen da ínsula, e é dividida pelo oblíquo sulco central da ínsula em uma porção anterior maior em área, e em uma porção posterior. Enquanto a porção anterior é composta em geral por três giros curtos que se originam no ápice da ínsula, que corresponde ao aspecto mais proeminente da pirâmide insular, com o mais anterior se extendendo sobre a superfície anterior da ínsula, a porção posterior é composta em geral por dois giros longos dispostos obliquamente e em paralelo. Do ápice da ínsula se originam também os giros transverso e acessório da ínsula, que em conjunto constituem o pólo da ínsula ${ }^{18,19}$. O giro transverso da ínsula, de disposição mais inferior, se dispõe ao longo do limen da ínsula conectando a sua superfície com o lóbulo orbital póstero-medial, que por sua vez é constituído pela porção posterior do giro orbital medial e pela porção medial do giro orbital posterior ${ }^{18,25}$ e que se dispõe anteriormente à estria olfatória lateral.

A superfície insular é delimitada perifericamente pelo sulco circular de Reil ${ }^{16}$, ou peri-insular ${ }^{17,18}$, que se mostra interrompido apenas ao nível do limen da ínsula em função da disposição do já mencionado giro transverso da ínsula. Dada a sua conformação triangular, o seu sulco circular ou peri-insular é usualmente dividido nos segmentos peri-insulares anterior, superior e inferior ${ }^{18}$, também denominados respectivamente de sulcos limitantes anterior, superior e inferior da ínsula ${ }^{13}$ (Fig 2). 
Para a melhor compreensão dos espaços periinsulares é importante destacar que, dado o fato da ínsula possuir uma superfície lateral e uma superfície anterior, enquanto as porções do sulco circular da ínsula denominadas de sulcos limitantes superior e inferior se caracterizam morfologicamente como verdadeiros sulcos que delimitam respectivamente as transições ou deflexões existentes entre a superfície insular lateral e os opérculos frontoparietal e temporal, o chamado sulco limitante anterior da ínsula é bem mais profundo e se caracteriza morfologicamente como uma verdadeira fissura ou espaço que separa a superfície anterior da ínsula da superfície posterior do giro orbital posterior.

Sob o córtex insular e a sua respectiva substância branca subcortical, também denominada de cápsula extrema, se dispõem a fina lâmina de substância cinzenta que constitui o claustro, e sob este o putame envolvido pelas suas cápsulas externa e interna. Enquanto a delgada cápsula externa é constituída por fibras que recobrem apenas a porção lateral do putame e que são desprovidas de maior importância funcional, a cápsula interna é composta pelas importantes fibras de projeção que se originam e que se destinam a todo o córtex cerebral. Além de encobrir medialmente todo o putame, a cápsula interna também se extende posterior e inferiormente ao núcleo lentiforme ${ }^{1,7,23}$.

Por ser constituída por fibras que se dispõem por sob todo o córtex cerebral superiormente, e o pedúnculo cerebral e o tálamo inferiormente, a cápsula interna possui a forma de um leque que se abre de baixo para cima. Superiormente as fibras da cápsula interna passam a constituir o chamado centro semioval ou coroa radiada, a partir de um limite arbitrário dado pelos aspectos mais superiores dos núcleos lentiforme e caudado. Inferiormente, as fibras originadas no córtex e que se destinam ao pedúnculo cerebral se dispõem progressivamente póstero-lateralmente ao tálamo 1,7,23. Dada a topografia basal e medial de cada tálamo em relação ao seu respectivo hemisfério cerebral, as fibras da cápsula interna que constituem as conexões tálamo-corticais, denominadas de radiações talâmicas, inferiormente se dispõem medialmente às fibras que vem a constituir o pedúnculo cerebral, e superiormente se abrem em leque, se embricando com as demais fibras e se dispondo na direção da área cortical a que se destinam.

Dadas as conformações arredondadas da cabeça do núcleo caudado e do tálamo, e a disposição anterior do primeiro em relação ao segundo, a cápsula interna que se dispõem lateralmente a essas estru- turas apresenta a forma de um $\mathrm{V}$ em cortes axiais, com o seu vértice disposto medialmente e entre as duas estruturas, e apontando para o forame interventricular de Monro aí situado. Tendo então como base a topografia das suas fibras, a cápsula interna divide-se em cinco partes: 1) o ramo anterior, situado entre o putame e a cabeça do núcleo caudado, 2) o joelho, situado no vértice existente entre a cabeça do núcleo caudado, putame e tálamo, portanto lateral e adjacente ao forame interventricular, 3) o ramo posterior, situado entre o putame e o tálamo, sobre o qual se dispõem o corpo do núcleo caudado, 4) a parte retrolentiforme, que se dispõem posteriormente ao putame, e 5) a parte sublentiforme, que se dispõem inferiormente ao putame (Quadro 1).

Uma vez que a área da superfície lateral da ínsula é maior do que a área do putame ${ }^{18}$, os seus sulcos limitantes anterior, superior e inferior já praticamente recobrem respectivamente a porção ântero-basal do ramo anterior da cápsula interna, as fibras que do opérculo frontoparietal se dirigem para ambos os ramos e joelho da cápsula interna, e as suas partes retro e sublentiformes que se extendem ao lobo temporal. Dada a conformação em $\mathrm{V}$ com vértice

Quadro 1. Principais fibras da cápsula interna.

\begin{tabular}{|c|c|}
\hline \multirow[t]{2}{*}{ Ramo anterior } & Fibras fronto-ponto-cerebelares \\
\hline & $\begin{array}{l}\text { Radiação talâmica anterior } \\
\text { (córtex frontal, cíngulo) }\end{array}$ \\
\hline \multirow[t]{2}{*}{ Joelho } & Fibras córtico-nucleares \\
\hline & $\begin{array}{l}\text { Radiação talâmica superior } \\
\text { (porção anterior) }\end{array}$ \\
\hline \multirow[t]{6}{*}{ Ramo posterior } & Fibras fronto-ponto-cerebelares \\
\hline & Fibras córtico-espinais \\
\hline & Fibras córtico-reticulares \\
\hline & Fibras córtico-rubras \\
\hline & Fibras pálido-talâmicas \\
\hline & $\begin{array}{l}\text { Radiação talâmica superior } \\
\text { (córtex pré-motor, motor e somato-sensitivo) }\end{array}$ \\
\hline \multirow{4}{*}{$\begin{array}{l}\text { Parte } \\
\text { retrolentiforme }\end{array}$} & Fibras parieto-ponto-cerebelares \\
\hline & Fibras occipito-ponto-cerebelares \\
\hline & $\begin{array}{l}\text { Radiação talâmica posterior } \\
\text { (córtex parietal, occipital e temporal, incluindo } \\
\text { as radiações auditiva e óptica) }\end{array}$ \\
\hline & $\begin{array}{l}\text { Conexões inter-parietais, occipitais e } \\
\text { pulvinares }\end{array}$ \\
\hline \multirow{2}{*}{$\begin{array}{l}\text { Parte } \\
\text { sublentiforme }\end{array}$} & Fibras temporo-ponto-cerebelares \\
\hline & $\begin{array}{l}\text { Radiação talâmica inferior } \\
\text { (córtex temporal, núcleo amigdalóide, } \\
\text { radiação auditiva) }\end{array}$ \\
\hline
\end{tabular}

Adaptado de Brodal ${ }^{1}$, Türe et al. $^{20}$, Williams e Warwick ${ }^{23}$, Yasargil ${ }^{25}$. 

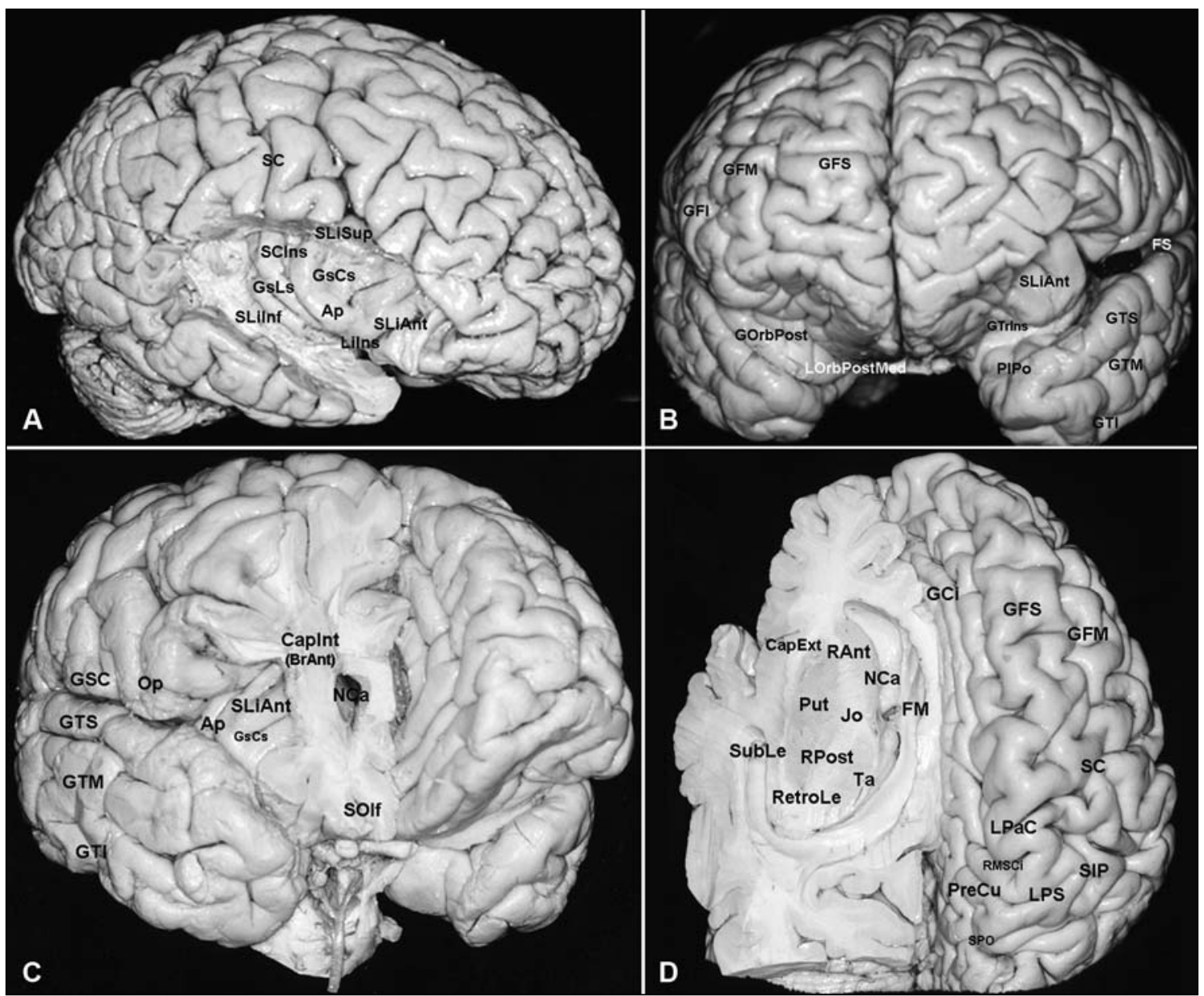

Fig 2. (A) A superfície lateral da ínsula: disposta sob os opérculos fronto-parietal e temporal (removidos nesta preparação), a superfície lateral da ínsula apresenta uma conformação piramidal com base triangular, caracterizada pelos seus giros curtos (GsCs) originados no seu ápice ( $A p$ ), e pelos seus giros longos (GsLs) dispostos posteriormente. (B) A superfície anterior da ínsula: além da superfície lateral, a ínsula possui também uma superfície anterior que se encontra encoberta pelo giro orbital posterior (GOrbPost). Dada a extensa área da superfície anterior da ínsula, o sulco limitante anterior da ínsula (SLiAnt) que se dispõe entre esta superfície e a superfície posterior do giro orbital (GOrbPost) é particularmente profundo e portanto se caracteriza como uma verdadeira fissura. (C) A profundidade do sulco limitante anterior (SLiAnt), se encontra separada da porção mais anterior do corno anterior ou frontal apenas pelas fibras do delgado ramo anterior da cápsula interna (Caplnt, RAnt). (D) A cápsula interna: a cápsula interna do núcleo lenticular é formada por cinco partes: o ramo anterior (RAnt) disposto entre o putame (Put) e a cabeça do núcleo caudado (NCa), o joelho (Jo) situado no vértice entre o putame (Put), a cabeça do núcleo caudado (NCa) e o tálamo (Ta), o ramo posterior (RPost) situado entre o putame (Put) e o tálamo (Ta) e o corpo do núcleo caudado (aqui não demonstrado) que se dispõem sobre o tálamo (Ta), a parte retrolenticular (RetroLe) e a parte sublenticular (SubLe), dispostas posterior e inferiormente ao putame (Put). Dada a conformação em $V$ das suas três partes mais mediais, o joelho da cápsula interna (Jo) se situa adjacentemente ao forame interventricular de Monro (FM). Ap: ápice da ínsula; CapExt: cápsula externa; Caplnt: cápsula interna; FM: forame interventricular de Monro; FS: fissura silviana; GCi: giro do cíngulo; GFI: giro fontal inferior; GFM: giro frontal médio; GFS: giro frontal superior; GOrbPost: giro orbital posterior; GSC: giro subcentral; GsCs: giros curtos da ínsula; GsLs: giros longos; GTI: giro temporal inferior; GTM: giro temporal médio; GTrIns: giro transverso da ínsula; GTS: giro temporal superior; Jo: joelho da cápsula interna; Lilns: limen da ínsula; LOrbPostMed: lóbulo orbital póstero-medial; LPaC: lóbulo paracentral; LPS: lóbulo parietal superior; NCa: núcleo caudado; Op: parte opercular do giro frontal inferior; PIPo: plano polar da superfície opercular temporal; PreCu: pré-cúneo; Put: putame; RAnt: ramo anterior da cápsula interna; RetroLe: parte retrolenticular da cápsula interna; RMSCi: ramo marginal do sulco do cíngulo; RPost: ramo posterior da cápsula interna; SC: sulco central; SCIns: sulco central da ínsula; SIP: sulco intraparietal; SLiAnt: sulco limitante anterior; SLilnf: sulco limitante inferior; SLiSup: sulco limitante superior; SOlf: sulco olfatório; SPO: sulco parieto-occipital; SubLe: parte sublentiforme da cápsula interna; Ta: tálamo. 
Quadro 2. Estruturas encefálicas que delimitam cada ventrículo lateral.

\begin{tabular}{|c|c|c|c|}
\hline \multirow{5}{*}{$\begin{array}{l}\text { Corno frontal } \\
\text { ou anterior }\end{array}$} & Assoalho & & Rostro do campo caloso \\
\hline & Parede anterior & & Joelho do corpo caloso \\
\hline & Teto & & Tronco do corpo caloso \\
\hline & Parede & Medial & Septo pelúcido \\
\hline & & Lateral & Cabeça do núcleo caudado \\
\hline \multirow{5}{*}{$\begin{array}{l}\text { Parte central ou } \\
\text { corpo ventricular }\end{array}$} & Assoalho & Medial & Corpo do fórnix \\
\hline & & Lateral & Face superior do tálamo \\
\hline & Teto & & Tronco do corpo caloso \\
\hline & Parede & Medial & Septo pelúcido \\
\hline & & Lateral & Corpo do núcleo caudado \\
\hline \multirow{6}{*}{$\begin{array}{l}\text { Átrio ou trígono } \\
\text { ventricular }\end{array}$} & Parede anterior & Medial & Crura do fórnix \\
\hline & & Lateral & Pulvinar do tálamo e cauda núcleo caudado \\
\hline & Teto & & Esplênio do corpo caloso \\
\hline & Parede & Lateral & Substância branca hemisférica/stratum sagital \\
\hline & & Medial & Hipocampo, bulbo do esplênio do corpo caloso \\
\hline & Assoalho & & Trígono colateral, esplênio do corpo caloso \\
\hline \multirow{7}{*}{$\begin{array}{l}\text { Corno temporal } \\
\text { ou inferior }\end{array}$} & Assoalho & Medial & Hipocampo \\
\hline & & Lateral & Eminência colateral \\
\hline & Teto & Medial & Cauda do núcleo caudado \\
\hline & & Lateral & Substância branca hemisférica, stratum sagital, amígdala \\
\hline & Parede & Anterior & Amígdala, substância branca hemisférica \\
\hline & & Medial & Fímbria do fórnix \\
\hline & & Lateral & Substância branca hemisférica/stratum sagital \\
\hline \multirow{4}{*}{$\begin{array}{l}\text { Corno occipital } \\
\text { ou posterior }\end{array}$} & Teto & & Esplênio do corpo caloso \\
\hline & Parede & Medial & Esplênio do corpo caloso, calcar avis \\
\hline & & Lateral & Esplênio do corpo caloso/stratum sagital \\
\hline & Assoalho & & Esplênio do corpo caloso \\
\hline
\end{tabular}

medial da cápsula interna, os locais de maior proximidade da superfície insular com as fibras da cápsula interna propriamente dita são justamente as áreas insulares que correspondem aos ângulos existentes entre os sulcos limitantes anterior e superior, e entre os sulcos limitante superior e inferior. Enquanto as fibras que constituem o ramo anterior da cápsula interna, situadas sob o ângulo insular mais anterior, carecem de maior importância funcional, as fibras capsulares dispostas sob o ângulo formado pelos sulcos limitantes superior e inferior, e sob todo o próprio sulco limitante inferior, se revestem de grande importância funcional uma vez que correspondem respectivamente às fibras córtico-espinhais motoras², à radiação talâmica superior, a radiação talâmica superior e às radiações auditiva e óptica ${ }^{3}$ que se continuam com o chamado stratum sagital (conjunto de fibras que constitui a parede lateral do corno temporal e do átrio ventriculares ${ }^{20}$. Dado que as fibras que compõem o stratum sagital constituem a parede lateral do átrio ventricular, e o teto e a parede lateral do corno inferior, o sulco limitante inferior se dispõem muito proximamente a estes compartimentos ventriculares (Fig 3).

Sob a porção mais anterior do sulco limitante inferior, entre o limen da ínsula e a parte mais anterior do corno inferior, e entre o limen da ínsula e o aspecto mais anterior da fissura corióidea, se dispõem um verdadeiro pedúnculo de fibras que conecta o lobo temporal ântero-medialmente à porção mais posterior da base frontal (fascículo uncinado, extensões ventrais e superior da amígdala, comissura anterior). Medialmente essas fibras se continuam com a região da substância inominada ${ }^{4,9,15}$ que corresponde à re- 

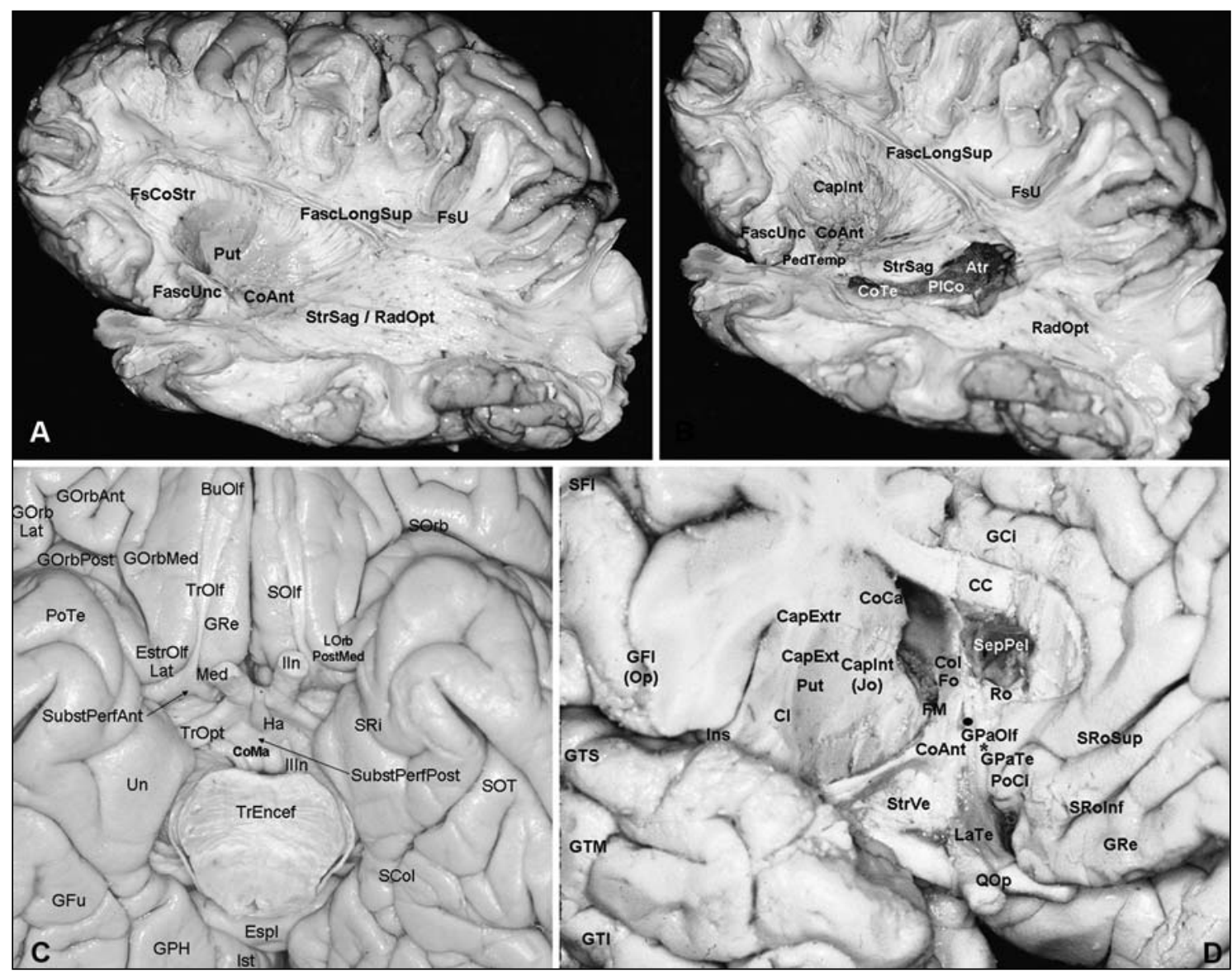

Fig 3. O stratum sagital e o pedúnculo temporal, e a região fronto-basal posterior (basal forebrain). (A) O stratum sagital (StrSag), aqui visualizado após a remoção do aspecto posterior do fascículo longitudinal superior (FascLongSup) que o recobre, corresponde à extensão lateral das partes retro e sublenticular da cápsula interna, e forma o teto e a parede lateral do átrio e do corno temporal. O seu principal contingente de fibras é constituído pela radiação óptica (RadOpt). (B) A remoção parcial do stratum sagital (StrSag) permite a observação da sua espessura e a exposição do átrio (Atr) e do corno temporal (CoTe). A remoção do putame permite a visualização das fibras que já compõem a cápsula interna (Caplnt), e dada a remoção conjunta da porção mais anterior do stratum sagital (StrSag), o pedúnculo temporal (PedTemp) pode aqui ser observado de forma bem individualizada. O pedúnculo temporal (PeTe) é delimitado anteriormente pelo limen da ínsula (aqui não demonstrado), e posteriormente pela cavidade ventricular (CoTe). (C) Sulcos e giros da porção frontotemporal da face inferior do cérebro, e substâncias perfuradas anterior e posterior: na superfície fronto-orbital, os giros orbitais anterior (GOrbAnt), posterior (GOrbPost), lateral (GOrbLat) e medial (GOrbMed) são separados pelo sulco orbital (SOrb) que apresenta a forma de um H. A área cortical fronto-basal denominada de substância perfurada anterior (SubstPerfAnt) tem como limites anteriores as estrias olfatórias lateral (StrOlfLat) e medial (StrOlfMed), como limite lateral o limen da ínsula (não visualizado nesta dissecção), como limite medial a fissura inter-hemisférica (FIH), e como limite posterior o trato óptico (TrOpt). Posteriormente a este último se dispõe a substância perfurada posterior (SubstPerfPost), que adentra a fossa interpeduncular. (D) A região estriatal-palidal ventral e a região septal: a região estriatal-palidal ventral, também denominada de striatum ventral (StrVe) e que se superpõe à região da chamada substância inominada, apesar de ser topograficamente mal delimitada dadas as suas continuidades com as estruturas adjacentes, corresponde basicamente à região fronto-basal que tem como parede anterior a substância perfurada anterior (removida nesta dissecção) e como parede posterior a comissura anterior (CoAnt). A região septal, ou subcalosa, se caracteriza pelos giros paraolfatório (GPaOlf) e paraterminal (GPaTe) que abrigam os núcleos septais, e se situa medialmente ao striatum ventral. Atr: átrio; BuOlf: bulbo olfatório; CapExt: cápsula externa; CapExtr: cápsula extrema Caplnt: cápsula interna; Caplnt(Jo): joelho da cápsula interna; CC: corpo caloso; Cl: claustro; CoAnt: comissura anterior; CoCa: corpo do núcleo caudado; ColFo: coluna do fórnix; CoMa: corpo mamilar; CoTe: corno temporal; Espl: esplênio do caloso; EstrOlfLat: estria olfatória lateral; EstrOlfMed: estria olfatória medial; FascLongSup: fascículo longitudinal superior; FascUnc: fascículo uncinado; FM: forame interventricular de Monro; FsCoStr: fibras córtico-estriatais; FsU: fibras em U; GCi: giro do cíngulo; GFI(Op): parte opercular do giro frontal inferior; GFu: giro fusiforme; GOrbAnt: giro orbital anterior; GOrbLat: giro orbital lateral; GOrbMed: giro orbital medial; GOrbPost: giro orbital posterior; GPaOlf: giro paraolfatório; GPaTe: giro paraterminal; GPH: giro parahipocampal; GRe: giro reto; GTI: giro temporal inferior; GTM: giro temporal médio; GTS: giro temporal superior; Ha: haste hipofisária; IIIn: nervo óculomotor; IIn: nervo óptico; In: ínsula; Ist: istmo do giro do cíngulo; LaTe: lâmina terminal; LOrbPostMed: lóbulo orbital pósteromedial; PedTemp: pedúnculo temporal; PICo: plexo corióideo; PoCi: pólo do cíngulo; PoTe: pólo temporal; Put: putame; QOp: quiasma óptico; RadOpt: radiação óptica; Ro: rostro do corpo caloso; SCol: sulco colateral; SepPel: septo pelúcido; SFI: sulco frontal inferior; SOIf: sulco olfatório; SOrb: sulco orbital; SOT: sulco occipitotemporal; SRi: sulco rinal; SRolnf: sulco rostral inferior; SRoSup: sulco rostral superior; StrSag: stratum sagital; StrVe: striatum ventral; SubstPerfAnt: substância perfurada anterior; SubstPerfPost: substância perfurada posterior; TrEnc: tronco encefálico (ponte); TrOlf: trato olfatório; TrOpt: trato óptico; Un: unco. 
Quadro 3. Estruturas encefálicas que constituem o III ventrículo.

\begin{tabular}{|c|c|c|}
\hline \multirow[t]{2}{*}{ Teto } & \multicolumn{2}{|c|}{ Corpos dos fórnices } \\
\hline & \multicolumn{2}{|c|}{$\begin{array}{l}\text { Cisterna do velum interpositum } \\
\text { (telas corióideas superior e inferior artérias } \\
\text { corióideas póstero-mediais, veias cerebrais } \\
\text { internas) }\end{array}$} \\
\hline \multirow[t]{3}{*}{$\begin{array}{l}\text { Paredes } \\
\text { laterais }\end{array}$} & \multicolumn{2}{|c|}{$\begin{array}{l}\text { Tálamos } \\
\text { (estria medular do tálamo) }\end{array}$} \\
\hline & \multicolumn{2}{|c|}{ Sulco hipotalâmico } \\
\hline & \multicolumn{2}{|l|}{ Hipotálamo } \\
\hline \multirow{4}{*}{$\begin{array}{l}\text { Parede } \\
\text { anterior }\end{array}$} & \multicolumn{2}{|c|}{ Pilares dos fórnices } \\
\hline & \multicolumn{2}{|c|}{ Comissura anterior } \\
\hline & \multicolumn{2}{|c|}{ Lâmina terminal } \\
\hline & \multicolumn{2}{|c|}{ Quiasma óptico } \\
\hline \multirow{4}{*}{$\begin{array}{l}\text { Parede } \\
\text { posterior }\end{array}$} & \multirow[t]{3}{*}{ Epitálamo } & Habênulas \\
\hline & & Comissura das habênulas \\
\hline & & Glândula pineal \\
\hline & \multicolumn{2}{|c|}{ Comissura posterior } \\
\hline \multirow[t]{6}{*}{ Assoalho } & \multirow[t]{4}{*}{ Hipotálamo } & Quiasma óptico \\
\hline & & $\begin{array}{l}\text { Eminência mediana/Haste } \\
\text { hipofisária }\end{array}$ \\
\hline & & Tuber cinéreo \\
\hline & & Corpos mamilares \\
\hline & \multirow[t]{2}{*}{ Mesencéfalo } & $\begin{array}{l}\text { Substância perfurada } \\
\text { posterior }\end{array}$ \\
\hline & & Tegmento mesencefálico \\
\hline
\end{tabular}

gião estriado-palidal ventral ${ }^{6,7}$, que é topograficamente delimitada anteriormente pela substância perfurada anterior, posteriormente pelas fibras que compõem a comissura anterior, e superiormente pelo ramo anterior da cápsula interna. Medialmente o striatum ventral por sua vez é contíguo à região septal que topograficamente já se dispõe sob a cabeça do núcleo caudado, e que é constituída pelos núcleos septais localizados internamente aos giros paraolfatórios e paraterminal.

Uma vez que medialmente o giro parahipocampal se encontra disposto sob o tálamo ao longo da fissura corióidea, é interessante frisar que além de se continuar posteriormente com os lobos parietal e occipital, o lobo temporal se encontra unido ao restante do hemisfério cerebral através das conexões dispostas sob o sulco limitante inferior acima descritas: ânteromedialmente pelo pedúnculo de fibras disposto sob o limen da ínsula, e póstero-lateralmente pelas fibras do stratum sagital, entre as quais se destacam as que compõem a radiação óptica. Este conjunto de fibras, que é usualmente denominado na literatura de língua inglesa de temporal stem, é então particularmente relacionado com a porção basal do bloco cerebral central.

As fibras da cápsula interna se dispõem portanto fundamentalmente internamente ao bloco cerebral central como que o dividindo, e ao transpor os seus limites morfologicamente se caracterizam como os principais pilares que mantém o bloco central incorporado ao restante do hemisfério cerebral: 1) a porção ântero-basal do ramo anterior, que se dispõem entre a profundidade do sulco limitante anterior e o recesso ventricular anterior à cabeça do núcleo caudado, o incorpora à região frontal anterior, 2) o restante do ramo anterior, o joelho e o ramo posterior, o incorporam superiormente à toda a convexidade frontoparietal, e 3) as partes retro e sublentiformes da cápsula interna, dispostas entre o núcleo lentiforme e o átrio e corno inferior, o incorporam ao corpo do lobo temporal. As fibras da parte retrolenticular (entre as quais se destaca a radiação auditiva que se destina ao giro de Heschl), e principalmente as fibras da parte sublentiforme da cápsula interna (entre as quais se destaca a radiação óptica que se destina ao córtex calcarino), lateralmente passam a compor o stratum sagital que recobre o átrio e o corno inferior ventriculares.

Ântero-basalmente, o conjunto de estruturas aqui descrito como constituindo o bloco cerebral central é contíguo à região fronto-basal mais posterior, usualmente denominada na literatura de língua inglesa de basal forebrain, que corresponde, de lateral para medial, à extensão medial do pedúnculo temporal, ao striatum ventral e à região septal.

Superiormente o bloco cerebral central é encoberto pelas fibras que compõem o corpo caloso e pela substância branca subcortical de cada hemisfério cerebral, e as suas superfícies medial, posterior e inferior se relacionam particularmente com as superfícies ventriculares supratentoriais (Quadros 2 e 3).

A superfície medial do bloco cerebral central é constituída anteriormente pelas superfícies intraventriculares da cabeça e do corpo do núcleo caudado, e pela superfície superior do tálamo, que afloram respectivamente no corno anterior e no corpo de cada ventrículo lateral, e póstero-inferiormente pelas superfícies talâmica e hipotalâmica que constituem a parede lateral do III ventrículo em cada hemisfério cerebral.

A superfície posterior propriamente dita do bloco cerebral central por sua vez é constituída pela super- 
fície do pulvinar talâmico, cuja porção superior aflora no átrio do ventrículo lateral e cuja porção inferior aflora nas cisternas ambiens e pineal ou quadrigêmina ${ }^{10,13,25}$.

Lateral e inferiormente a base do bloco central é delimitada pela asa lateral da cisterna ambiens ${ }^{24,25}$ que se dispõem na fissura transversa do cérebro, entre o pulvinar talâmico superiormente e o subículo do giro parahipocampal inferiormente.

\section{REFERÊNCIAS}

1. Brodal A. Neurological anatomy in relation to clinical medicine. 3.Ed New York: Oxford University Press, 1981.

2. Ebeling U, Reulen HJ. Subcortical topography and proportions of the pyramidal tract. Acta Neurochir (Wien) 1992;118:164-171.

3. Ebeling U, Von Cramon D. Topography of the uncinate fascicle and adjacent temporal fiber tracts. Acta Neurochir (Wien) 1992;115:143-148.

4. Federative Committee on Anatomical Terminology. International anatomical terminology. Stuttgart: Thieme, 1998.

5. Ferreira AG. Dicionário de latim-português. Porto: Porto Editora, 1966.

6. Heimer L. A new anatomical framework for neuropsychiatric disorders and drug abuse. Am J Psychiatry 2003;160:1726-1739.

7. Heimer L. The human brain and spinal cord. 2.Ed. New York: Springer Verlag, 1995.

8. Lavyne MH, Patterson RH. The subchoroidal trans-velum interpositum approach. In Apuzzo MLJ (Ed). Surgery of the third ventricle. 2.Ed. Baltimore: Williams and Wilkins, 1988:453-470.

9. Lockard I. Desk reference for neuroanatomy: a guide to essential terms. New York: Springer-Verlag, 1977.

10. Meneses MS. Neuroanatomia aplicada. Rio de Janeiro: GuanabaraKoogan, 1999.

11. Nagata S, Rhoton AL Jr., Barry M. Microsurgical anatomy of the choroidal fissure. Surg Neurol 1988;30:3-59.
12. Ono M, Kubik S, Abernathey CD. Atlas of cerebral sulci. Stuttgart: Thieme, 1990.

13. Rhoton AL Jr. Cranial anatomy and surgical approaches. Neurosurgery 2003;53:1-746.

14. Ribas GC. [Comment of: Siwanuwatn R, Deshmukh P, Zabramski JM, Preul MC, Spetzler RF. Microsurgical anatomy and quantitative analysis of the transtemporal-transchoroidal fissure approach to the ambient cistern. Neurosurgery 2005;57(Operative Neurosurgery Suppl 4):ONS233-234.

15. Sociedade Brasileira de Anatomia. Terminologia anatômica. São Paulo: Manole, 2001.

16. Taveras JM, Wood EH. Diagnostic neuroradiology. 2.Ed. Baltimore: Williams and Wilkins, 1976

17. Testut L, Latarjet A. Tratado de anatomia humana. 8.Ed. Barcelona: Salvat, 1932.

18. Türe U, Yasargil DCH, Al-Mefty O, Yasargil MG. Topographic anatomy of the insular region. J Neurosurg 1999;90:730-733.

19. Türe U, Yasargil MG, Al-Mefty O, Yasargil DCH. Arteries of the ínsula. J Neurosurg 2000;92:676-687.

20. Türe U, Yasargil MG, Friedman AH, Al-Mefty O. Fiber dissection technique: lateral aspect of the brain. Neurosurgery 2000;47:417-427.

21. Wen HT, Rhoton AL Jr, Oliveira E. Transchoroidal approach to the third ventricle: an anatomic study of the choroidal fissure and its clinical application. Neurosurgery 1998;42:1205-1219.

22. Wen HT, Rhoton AL Jr, Oliveira E, et al. Microsurgical anatomy of the temporal lobe: Part I: mesial temporal lobe anatomy and its vascular relationships and applied to amygdalohippocampectomy. Neurosurgery 1999;45:549-592.

23. Williams PL, Warwick R, editors. Gray's anatomy. 36.Ed. Philadelphia: Saunders, 1980

24. Yasargil MG. Microneurosurgery. Stuttgart: Georg Thieme, 1984. v.I.

25. Yasargil MG. Microneurosurgery. Stuttgart: Georg Thieme, 1994. v.IVa.

26. Yasargil MG, Teddy PJ, Roth P. Selective amygdalo-hippocampectomy: operative anatomy and surgical technique. Adv Tech Stand Neurosurg 1985;12:93-123.

27. Yasargil MG, Krisht AF, Türe U, Al-Mefty O, Yasargil DCH. Microsurgery of insular gliomas: Part I: surgical anatomy of the Sylvian cistern. Contemporary Neurosurgery 2002;24:1-8. 